\title{
Transplantation of bone marrow-derived endothelial progenitor cells overexpressing Delta-like-4 enhances functional neovascularization in ischemic myocardium
}

\author{
HONG HUANG $^{1}$, FENG HUANG ${ }^{2}$ and JIAN-PING HUANG ${ }^{3}$ \\ Departments of ${ }^{1}$ Cardiothoracic Surgery and ${ }^{2}$ Emergency, The First People's Hospital of Nanning, Nanning, Guangxi 530022; \\ ${ }^{3}$ Alibaba Business College, Hangzhou Normal University, Hangzhou, Zhejiang 310036, P.R. China
}

Received April 11, 2013; Accepted August 12, 2013

DOI: $10.3892 / \mathrm{mmr} .2013 .1657$

\begin{abstract}
Delta-like-4 (Dll-4) prevents excess angiogenic sprouting and promotes the formation of a well-differentiated vascular network. Therefore, transplantation of Dll-4-overexpressing endothelial progenitor cells (EPCs) was hypothesized to be superior to transplantation of EPCs in the treatment of ischemic heart disease. In the current study, EPCs harvested from C57BL/6 mouse bone marrow were infected in vitro with Dll-4 (EPC $\left.{ }^{\text {Dll-4+}}\right)$ or Dll-4 knockdown (EPC ${ }^{\text {Dll-4-) }}$ with recombinant lentiviral vectors and the control cells were non-transfected or transduced with mock vectors $\left(\mathrm{EPC}^{\text {null }}\right)$. Eight-week-old C57BL/6 mice underwent ligation of the left anterior descending artery to establish a myocardial infarction (MI) model. The ligated animals were randomly divided into 5 groups, which, following one week, were intravenously injected with EPCs, EPC ${ }^{\text {null }}, \mathrm{EPC}^{\text {Dll-4+ }}$ EPC $^{\text {Dll-4- }}$ or medium. Two weeks later, echocardiographic assessment, western
\end{abstract}

Correspondence to: Dr Feng Huang, Department of Emergency, The First People's Hospital of Nanning, 89 Qixing Road, Nanning, Guangxi 530022, P.R. China

E-mail: huangfeng3000@126.com

Dr Jian-Ping Huang, Alibaba Business College, Hangzhou Normal University, 16 Xuelin Street, Hangzhou, Zhejiang 310036, P.R. China E-mail: hjp@hznu.edu.cn

Abbreviations: EPCs, endothelial progenitor cells; Dll, Delta-like; Jag, Jagged; NICD, Notch intracellular domain; bHLH, basic helix-loop-helix; Hes, hairy/enhancer of split; Hey, Hes-related protein; ECs, endothelial cells; PBS, phosphate-buffered saline; VEGF, vascular endothelial growth factor; shRNAs, short hairpin RNAs; MTT, 3-(4,5-dimethylthiazol-2-yl)-2,5-diphenyltetrazolium bromide; MI, myocardial infarction; LV, left ventricular; LVID, left ventricular internal dimensions; LVIDD, LVID in diastole; LVIDS, LVID in systole; EF, ejection fraction; FS, fractional shortening; ANOVA, analysis of variance; mTOR, mammalian target of rapamycin

Key words: endothelial progenitor cells, Delta-like-4, transplantation, angiogenesis, tubulogenesis, ischemic heart disease blotting, fluorescent microsphere and histological studies were performed. The results demonstrated that the number of mature vessels and blood flow in ischemic myocardium were increased in the EPC ${ }^{\text {Dll-4+ }}$ group, but were markedly decreased in the EPC ${ }^{\text {Dll-4- }}$ group compared with the control groups. The expression levels of Dll-4, hairy/enhancer of split (Hes)-related protein 1 (Hey-1), phosphorylation of mammalian target of rapamycin (mTOR) and p70S6 kinase (p70S6K) were significantly increased in the EPC ${ }^{\text {Dll-4+ }}$ group, while they were markedly decreased in the EPC ${ }^{\text {Dll-4- }}$ group. Furthermore, for $\mathrm{EPC}^{\mathrm{Dll}-4+}$-treated animals, an enhanced cardiac function was observed as assessed by echocardiography. Thus, the transplantation of Dll-4-overexpressing EPCs stimulates neovascularization effectively, increases the blood flow to the ischemic zone and improves cardiac function. These effects may be due to the activation of Notch/Hey-1/mTOR/p70S6K signaling pathways, which are initiated by Dll-4.

\section{Introduction}

The formation of new blood vessels is an important process required for healing wounds and for restoring blood flow to tissue following injury or insult (1). A number of stem/progenitor cells, including bone marrow-derived endothelial progenitor cells (EPCs) and mononuclear cell, have been identified for their pro-angiogenic potential to improve perfusion as an experimental or clinical option $(2,3)$. As precursors of mature endothelial cells (ECs), EPCs are capable of increasing the neovascularization of ischemic tissue and delaying the initiation and progression of coronary artery disease $(4,5)$. Circulating EPCs were found to exhibit similar features of ECs, possess the ability to home to sites of ischemia and contribute to the formation of new blood vessels (6). Thus, EPCs have been used as seeding cells in tissue engineering and stem cell therapy.

Increasing evidence has demonstrated that the Notch signaling pathway is one of the most important mechanisms involved in the regulation of neovascularization. In mammals, the Notch signaling pathway includes five transmembrane ligands, i.e., Delta-like (D1l)-1, Dll-3, Dll-4, Jagged (Jag)-1 and Jag-2 and four transmembrane receptors, i.e., Notch-1, $-2,-3$ and -4 . The interaction between the Notch receptor and 
ligand leads to cleavage of the Notch intracellular domain (NICD) and translocation to the nucleus, thereby activating downstream target genes, including basic helix-loop-helix (bHLH) proteins which include hairy/enhancer of split (Hes) and Hes-related protein (Hey) $(7,8)$. The Notch ligand Dll-4 has been identified as a promising new target for angiogenesis in preclinical studies. For example, the balance between sprout and tube formation is established as being important for the generation of a new functional vessel, which is hypothesized to be modulated by Dll-4 from tip ECs to neighboring stalk ECs in order to restrict the emergence of excessive sprout through the repression of vascular endothelial growth factor (VEGF) receptor 2 (VEGFR2) transcription and consequently the reduction of responsiveness to $\operatorname{VEGF}(9,10)$. By contrast, the inhibition of Dll-4-mediated signaling is hypothesized to retard tumor growth, despite an increase of tumor vasculature density since the established vascular network is functionally inefficient (11-13), suggesting that Dll-4 attenuates the formation of ineffective vascular branch and promotes the remainder to form functional vessels. Thus, Dll-4-overexpressing EPCs were hypothesized to be superior to untreated EPCs in the formation of productive blood vessels in ischemic myocardium.

\section{Materials and methods}

Animal ethics. All procedures were performed in compliance with the guidelines for the Care and Use of Laboratory Animals published by the National Institutes of Health (NIH Publication No. 85-23, revised 1996) and approved by the Animal Care and Use Committee of the First People's Hospital of Nanning, China.

Isolation, cultivation and identification of EPCs. Two 6-week-old C57BL/6 mice were sacrificed by cervical dislocation. Following removal of the tips of the hind legs and vertebrae, bone marrow was collected by flushing out the content of femurs and tibias with PBS. Mononuclear cells were collected from the bone marrow by density gradient centrifugation using Histopaque 1077 (Sigma-Aldich, St. Louis, MO, USA) according to the manufacturer's instructions. The isolated cells were cultivated in dishes coated with fibronectin (R\&D Systems, Minneapolis, MN, USA) and induced by EBM-2 Single Quots (Lonza, Basel, Switzerland) with supplements at $37^{\circ} \mathrm{C}$ with $5 \% \mathrm{CO}_{2}$ in humidified air at a density of $5 \times 10^{6}$ cells $/ \mathrm{cm}^{2}$. Following 3 days in culture, non-adherent cells were removed by washing with PBS, new medium was applied and the cultivation was maintained for 7 days. Immunofluorescence staining and flow cytometry were used to identify EPCs, which was performed as previously described by Liu et al (14).

Recombinant lentiviral vector construction and cell infection. The procedures for recombinant lentiviral vector construction, cell infection and selection of the stable cell line were performed as previously described by Chen and Zhou (15). In brief, to produce Dll-4 recombinant lentiviral vectors, the plasmids pAJ-Ubi-eGFP-3Flag, psPAX2 (gag/pol element) and pMD2.G (VSVG element) (Auragene Bioscience Inc., Changsha, China) were used according to the manufacturer's instructions. Following lentiviral vector infection, the stable cell line overexpressing Dll-4 was cultured in a $5 \% \mathrm{CO}_{2}$-humidified incubator at $37^{\circ} \mathrm{C}$. EPCs infected with Dll-4 recombinant lentiviral vectors were labeled $\mathrm{EPC}^{\mathrm{DIl}-4+}$ and EPCs infected with mock vectors were labeled EPC ${ }^{\text {null }}$

To determine whether transplantation of Dll-4-inhibited EPCs had an opposed effect on neovascularization, EPCs were infected with lentiviral constructs encoding short hairpin RNA (shRNA) against Dll-4. The plasmids pAJ-U6-shRNA-CMV-Puro/eGFP, psPAX2 and pMD2.G (Auragene Bioscience) were transfected into 293T cells according to the instructions for Lipofectamine 2000 (Invitrogen Life Technologies, Carlsbad, CA, USA). Following a 48-h transduction, infected EPCs were selected to generate a stable Dll-4-shRNA line. EPCs transfected with recombinant lentiviral vectors encoding Dll-4-shRNA were labled EPC ${ }^{\text {Dll-4- }}$.

Quantitative PCR ( $q P C R$ ) was used to identify the efficiency of gene transfection. Total RNA was extracted from each sample with TRIzol reagent (Invitrogen Life Technologies) and reversed transcribed into first-strand cDNA by RevertAid ${ }^{\mathrm{TM}}$ First Strand cDNA Synthesis kit (Fermentas, Vilnius, Lithuania) according to the manufacturer's instructions. The synthesized cDNA was used for qPCR analysis of Dll-4 mRNA expression with SYBR ${ }^{\circledR}$ Premix Ex Taq ${ }^{\mathrm{TM}}$ (Takara Bio, Inc., Shiga, Japan). The sense sequence of Dll-4 primers was 5'-CGAGGGAACAGAGTTGAGGAGT-3' and the antisense sequence was 5'-AATACAGATGCCCACAGGAGC-3'. Fluorescence qPCR was performed on the ABI PRISM 7300 SDS apparatus (Applied Biosystems, Foster City, CA, USA).

Western blot analysis. Each sample was lysed in $0.2 \mathrm{ml}$ lysis buffer (Calbiochem, La Jolla, CA, USA). The protein concentrations were determined by the Bradford method (Bio-Rad, Hercules, CA, USA). Total protein $(20 \mu \mathrm{g})$ was separated on $10 \%$ SDS-PAGE gels and transferred to PVDF membranes (Millipore, Billerica, MA, USA) using the semi-dry transfer method. Membranes were blocked for $1 \mathrm{~h}$ in Tris-buffered saline containing $0.01 \%$ Tween-20 with $10 \%$ non-fat dried milk and incubated overnight at $4{ }^{\circ} \mathrm{C}$ with the relevant antibodies: Dll-4 and Hey-1 antibody (Santa Cruz Biotechnology, Inc., Santa Cruz, CA, USA), mammalian target of rapamycin (mTOR) and phospho-mTOR (Ser2448) antibody (Abcam, Cambridge, UK), p70S6 kinase (p70S6K) and phospho-p70S6K (Thr421/Ser424) antibody (Cell Signaling Technology, Inc., Danvers, MA, USA). Membranes were rinsed and incubated for $1 \mathrm{~h}$ with the corresponding peroxidase-conjugated secondary antibodies. Chemiluminescent detection was performed using the ECL kit (Pierce Biotechnology, Inc., Rockford, IL, USA). All bands were analysed using Image J software (version 1.6 NIH).

In vitro angiogenesis assay. Tubulogenesis was induced using an in vitro Angiogenesis Assay kit (\#ECM625, Millipore) following the manufacturer's instructions (16). Briefly, ECMatrix $^{\mathrm{TM}}$ solution was thawed on ice overnight, mixed with 10X ECMatrix ${ }^{\mathrm{TM}}$ diluents and placed in a 96-well tissue culture plate at $37^{\circ} \mathrm{C}$ for $1 \mathrm{~h}$ to allow the matrix solution to solidify. EPCs $\left(1 \times 10^{4}\right.$ cells/well) in $50 \mu \mathrm{l}$ of medium were cultured on the top of the solidified matrix solution. Following $18 \mathrm{~h}$ of 
incubation at $37^{\circ} \mathrm{C}$, the tubule formation was inspected under an inverted light microscope at x200 magnification. Tubule formation was defined as a structure exhibiting a length four times its width. Five random microscopic fields were assessed for each well and the average number and the total length of tubules/200x field was determined and compared with the control cells.

Assessment of cell death induced by $\mathrm{H}_{2} \mathrm{O}_{2}$. The viability of EPCs was determined by a 3-(4,5-dimethylthiazol-2-yl)-2,5-diphenyltetrazolium bromide (MTT) (Sigma-Aldrich) assay. A total of $1 \times 10^{4}$ cells were equally seeded into each well in 96-well microplates. Following incubation with $\mathrm{H}_{2} \mathrm{O}_{2}$ for $1 \mathrm{~h}$, the medium was replaced with MTT solution $(0.5 \mathrm{mg} / \mathrm{ml}$ in PBS). Incubation was continued for $4 \mathrm{~h}$ and then the supernatant was gently removed. Dimethyl sulfoxide (DMSO) (Sigma-Aldrich) was added and the absorbance was read at $490 \mathrm{~nm}$ on a spectrophotometer (Bio-Rad) and the percentage of cell viability was obtained.

Creation of myocardial infarction (MI) model and cell trans-

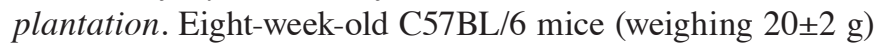
underwent ligation of the left coronary artery to produce MI. Following anesthetization, the animals were orally intubated with a $1.0-\mathrm{mm}$ OD intubation cannula and connected to a small animal volume-control ventilator (HES-HA MiniVent 845, Harvard Apparatus, Holliston, MA, USA). The left anterior descending artery was ligated 2-3 $\mathrm{mm}$ from its origin between the pulmonary artery conus and the left atrium using 8-0 sutures. The ligated animals were monitered by electrocardiograph using the RM6240BD system (Chengdu Instrument Company, Chengdu, China) to determine MI models. One week following coronary ligation, the surviving mice were randomly subdivided into 5 groups (PBS, EPCs, EPC null, $\mathrm{EPC}^{\mathrm{Dll}-4+}$ and $\mathrm{EPC}^{\mathrm{Dll}-4-} ; 20$ animals in each) and administered with intravenous injection of PBS, EPCs, $\mathrm{EPC}^{\text {null }}, \mathrm{EPC}^{\mathrm{Dll}-4-}$ and $\mathrm{EPC}^{\mathrm{Dll}-4+}$ in the tail vein. Each animal received an injection of $5 \times 10^{6}$ cells $/ 100 \mu 1$ in PBS or PBS alone with a total volume of $50 \mu 1$.

Assessment of cardiac function by echocardiography. A transthoracic echocardiographic study was performed by an experienced blinded cardiologist at 14 days post-transplantation using an echocardiographic system (SONOS 5500, Hewlett-Packard, Andover, MA, USA) equipped with a $15.0 \mathrm{MHz}$ transducer. For analysis of left ventricular (LV) function, left ventricular internal dimensions (LVID) were measured at diastole (LVIDD) and systole (LVIDS). After two-dimensional images were obtained, the M-mode cursor was positioned to the parasternal long axis view at the papillary muscle level. LV ejection fraction (EF) and fractional shortening (FS) were calculated as follows: $\mathrm{EF}(\%)=\left(\mathrm{LVIDD}^{3}-\mathrm{LVIDS}^{3}\right) / \mathrm{LVIDD}^{3} \times 100 \%$ and $\mathrm{FS}(\%)$ $=[($ LVIDD - LVIDS $) /$ LVIDD $] \times 100 \%$, respectively. Each parameter was measured from a minimum of 3 consecutive beat cycles in each image.

Myocardial blood flow. Measurement of blood flow to the peri-infarcted and infarcted area of mouse hearts was performed as previously described (17).
Capillary density. To identify mature capillary density, the tissue sections $(5 \mu \mathrm{m})$ of the infarcted zone were stained with anti-VIII factor antibody (Santa Cruz Biotechnology, Inc.). Immunohistochemical staining was performed as previously described (17).

Statistical analysis. Data are presented as the mean \pm SD. A method of ANOVA (analysis of variance) with Scheffe's post-hoc test was used to identify differences among the groups. $\mathrm{P}<0.05$ was considered to indicate a statistically significant difference.

\section{Results}

Efficiency of gene transfection and alteration of relative signals. qPCR data indicated that Dll-4 gene expression in $\mathrm{EPC}^{\mathrm{Dll}-4+}$ was 17-fold higher compared with the expression in EPCs. The D1l-4 mRNA level was $~ 65.4 \%$ lower in EPC $^{\text {Dll-4- }}$ compared with EPCs. Western blot analysis showed that the expression of Dll-4, Hey-1, phospho-mTOR and phospho-p70S6K were markedly increased in $\mathrm{EPC}^{\mathrm{Dll}-4+}$ and markedly decreased in EPC ${ }^{\text {Dll-4- }}$ (Fig. 1). Furthermore, a similar alteration was also detected in the EPCs-, EPC ${ }^{\text {null }}$, EPC $\mathrm{EPIl}^{\mathrm{DH}}$ and $\mathrm{EPC}^{\mathrm{DIl}-4-}$-treated animals when the peri-infarcted and infarcted heart tissue was extracted for western blot analysis. These observations suggested that the overexpression of Dll-4 results in the increase of its downstream target molecule, Hey-1 and thus, the activation of mTOR signaling pathway.

Protective effect of Dll-4 on the $\mathrm{H}_{2} \mathrm{O}_{2}$-injured EPCs. To determine the effect of Dll-4 on the viability of $\mathrm{H}_{2} \mathrm{O}_{2}$-treated EPCs, the cells of each group were exposed to increasing concentrations of $\mathrm{H}_{2} \mathrm{O}_{2}$ from 0 to $1,000 \mu \mathrm{mol} / \mathrm{l}$ (data from the pilot study were not shown). The previous study showed that $150 \mu \mathrm{mol} / 1$ $\mathrm{H}_{2} \mathrm{O}_{2}$ is the optimal concentration. Dll-4-overexpressing EPCs were more resistant to $\mathrm{H}_{2} \mathrm{O}_{2}$ compared with EPCs or EPC null following incubation with the apoptotic stimulus $(\mathrm{P}<0.01)$. By contrast, Dll-4-inhibited EPCs were more readily damaged by $\mathrm{H}_{2} \mathrm{O}_{2}$ (Fig. 2).

Capillary-like tube formation. EPCs were seeded on a solidified matrix and incubated with collected medium for $18 \mathrm{~h}$. Quantification of branch points/200x microscopic field demonstrated that the number of tubular structures in the EPC $\mathrm{Ell}^{\mathrm{l}+}$ group was higher compared with the EPC and EPC ${ }^{\text {null }}$ groups. Tubules in the $\mathrm{EPC}^{\text {Dll-4+ }}$ group were qualitatively different and more complex compared with those in the control wells. Despite the number of emerging sprouts being greater in the

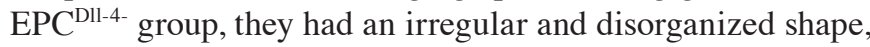
suggesting that knockdown of the Dll-4 gene impacts on EPCs ability to form functional tubules (Fig. 3).

Improvement of LV contractile function. To assess cardiac function, echocardiographic studies were performed 2 weeks post-transplantation. EF and FS were improved in the EPCs, $\mathrm{EPC}^{\text {null }}$ and $\mathrm{EPC}^{\mathrm{Dll}-4+}$ groups when compared with the PBS group $(\mathrm{P}<0.01$ or $\mathrm{P}<0.001)$. Furthermore, EF and FS were higher in the EPC ${ }^{\text {Dll-4+ }}$ group compared with the EPCs and $\mathrm{EPC}^{\text {null }}$ groups $(\mathrm{P}<0.05$; Fig. 4). However, there was no statistical significance in enhancing cardiac function between 
A

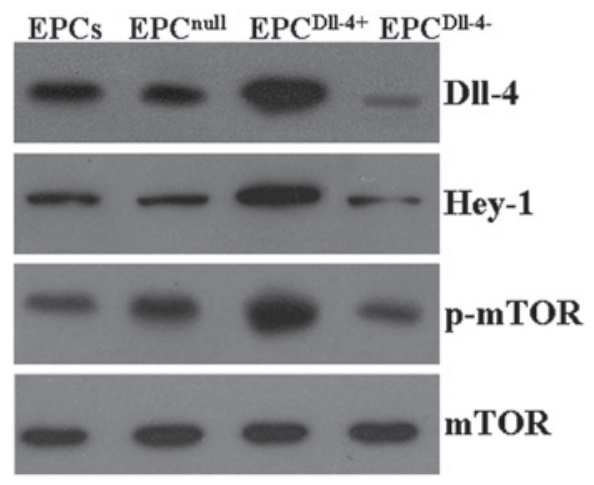

B

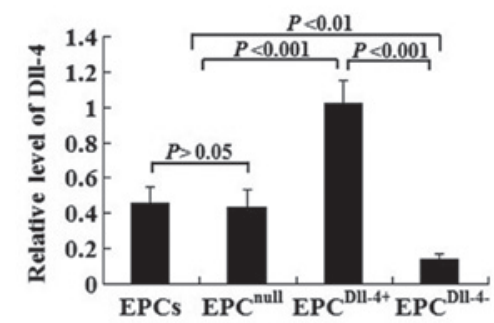

D

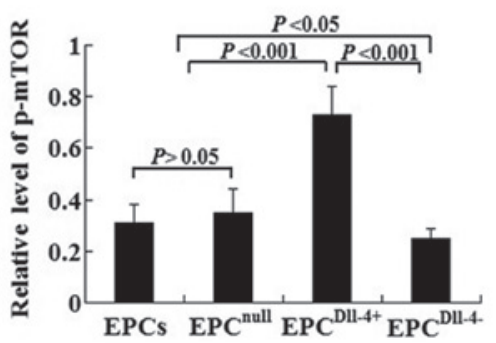

EPCs EPC $^{\text {null }}$ EPC $^{\text {Dll-4+ }}$ EPC $^{\text {Dll-4- }}$

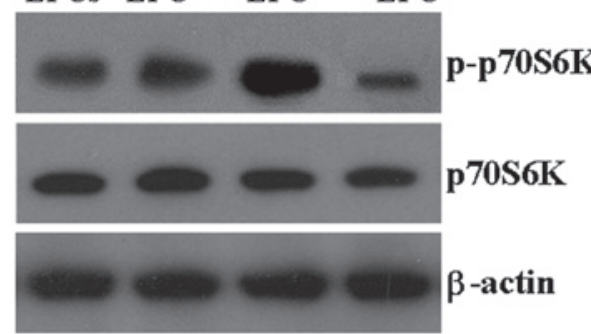

C

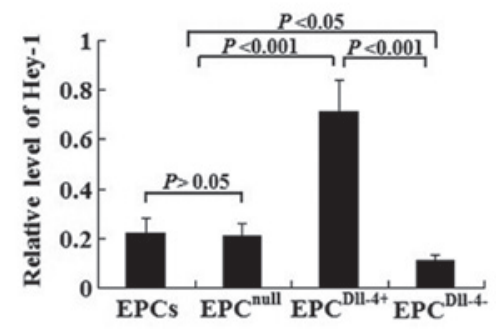

$\mathbf{E}$

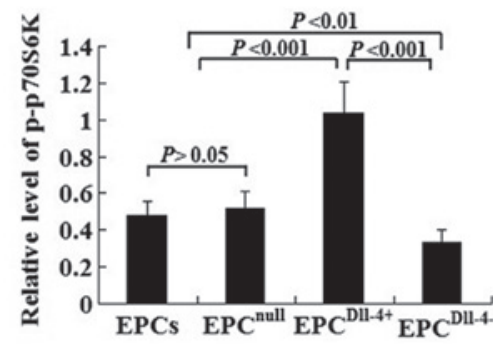

Figure 1. Western blot analysis of Dll-4 expression and alteration of the downstream signaling pathways in vitro. (A-E) Data show that the expression of Dll-4, Hey-1, one of the downstream of Notch signaling pathways and the phosphorylated status of mTOR and p70S6K, was significantly increased in EPC ${ }^{\text {Dll-4+ }}$ and markedly decreased in EPC ${ }^{\text {Dll-4- }}$. EPCs, endothelial progenitor cells; Dll-4, Delta-like-4; Hey-1, Hes-related protein 1; mTOR, mammalian target of rapamycin, p70S6K, p70S6 kinase; EPC ${ }^{\text {null }}$, EPCs infected with mock vectors; EPC ${ }^{\text {Dll-4+ }}$, Dll-4-overexpressing EPCs; EPC ${ }^{\text {Dll-4- }}$, Dll-4-inhibited EPCs.

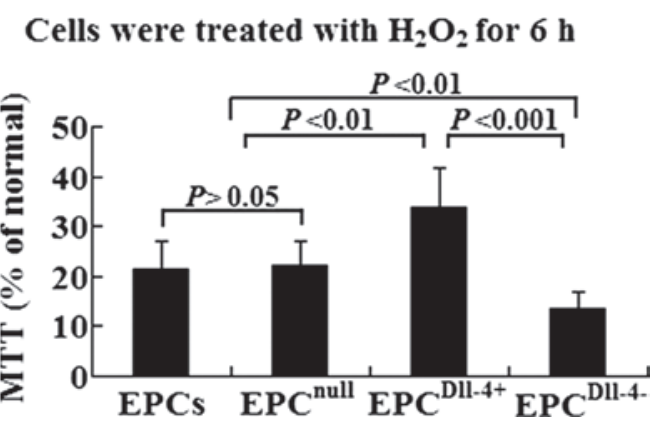

Figure 2. Analysis of cell viability using MTT assay in EPCs, EPC null, $\mathrm{EPC}^{\text {DIl-4+ }}$ and $\mathrm{EPC}^{\text {Dll-4- }}$ following incubation with $\mathrm{H}_{2} \mathrm{O}_{2}$ for 6 h. EPC ${ }^{\text {Dll-4+ }}$ showed a higher resistance against hypoxia when compared with the control cells. MTT, 3-(4,5-dimethylthiazol-2-yl)-2,5-diphenyltetrazolium bromide; D11-4, Delta-like-4; EPCs, endothelial progenitor cells; EPC null, EPCs infected with mock vectors; $\mathrm{EPC}^{\text {Dll-4+ }}$, Dll-4-overexpressing EPCs; $\mathrm{EPC}^{\text {Dll-4- }}$ Dll-4-inhibited EPCs.

EPC ${ }^{\text {Dll-4- }}$ and PBS therapy $(\mathrm{P}>0.05)$. These results indicate that transplantation of the Dll-4-overexpressing EPCs had an improved therapeutic effect for improving the LV function of MI animals compared with transplantation of untreated EPCs. By contrast, pre-inhibition of Dll-4 in the transplanted cells resulted in a poor effect.

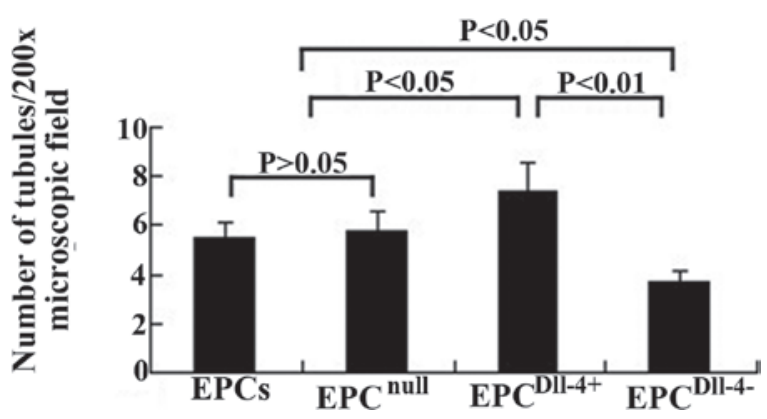

Figure 3. In vitro angiogenesis assay of EPC tubule formative capacity. The number of tubular structures was higher in the $\mathrm{EPC}^{\mathrm{Dll}-4+}$ group compared with the EPCs and EPC ${ }^{\text {null }}$ groups. However, the ability of EPCs to form mature vessels was attenuated evidently while inhibiting the intracellular Dll-4 gene. Dll-4, Delta-like-4; EPCs, endothelial progenitor cells; EPC ${ }^{\text {null }}$ EPCs infected with mock vectors; $\mathrm{EPC}^{\mathrm{Dll}-4+}$, Dll-4-overexpressing EPCs; $\mathrm{EPC}^{\text {DIl-4- }}$, Dll-4-inhibited EPCs.

Transplantation of Dll-4-overexpressing EPCs increases to form mature micovessels in ischemic myocardium. Semiquantitative analysis showed that, 2 weeks following cell transplantation, the number of blood vessels in peri-infarcted and infarcted tissue was significantly increased in the EPC $(23.1 \pm 4.8)$ and $\mathrm{EPC}^{\text {null }}(22.6 \pm 5.2)$ groups when compared 


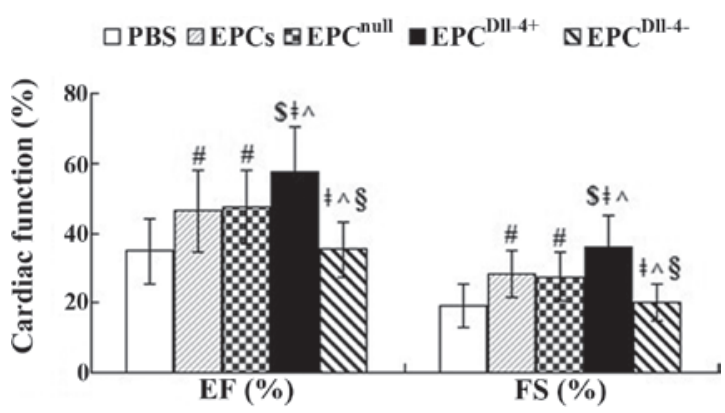

Figure 4. Improvement of LV contractile function. The LV, EF and FS were compared among these groups 2 weeks post-transplantation. Dll-4, Delta-like-4; LV, left ventricular, EF, ejection fraction; FS, ejection fraction; EPCs, endothelial progenitor cells; EPC ${ }^{\text {null }}$, EPCs infected with mock vectors; EPC $^{\text {Dll-4+ }}$, Delta-like-4 (Dll-4)-overexpressing EPCs; EPC ${ }^{\text {Dll-4- }}$, Dll-4-inhibited EPCs; ${ }^{~} \mathrm{P}<0.01$ vs. $\mathrm{PBS}$ group; ${ }^{\mathrm{P}} \mathrm{P}<0.001$ vs. $\mathrm{PBS}$ group; ${ }^{\ddagger} \mathrm{P}<0.01$ vs. $\mathrm{EPCs}$ group; ${ }^{\wedge} \mathrm{P}<0.01$ vs. $\mathrm{EPC}^{\text {null }}$ group; ${ }^{\circledR} \mathrm{P}<0.001$ vs. $\mathrm{EPCD}{ }^{11-4+}$ group group.

with the PBS group $(13.2 \pm 2.4)(\mathrm{P}<0.01)$. The microvessel number was further increased in the EPC ${ }^{\text {Dll-4+ }}$ group and an increase in immature vessel proliferation was observed in the peri-infarcted and infarcted area of mouse heart 2 weeks following treatment with $\mathrm{EPC}^{\text {DIl-4- }}$, however, mature vessels with a lumen were barely detected (Fig. 5). This indicated that Dll-4 may contribute to angiogenesis and tubulogenesis in ischemic myocardium.

Treatment of Dll-4-overexpressing EPCs increases the blood flow in ischemic myocardium. To determine whether new blood vessels translate to increased coronary blood flow to the infarcted myocardium, functional microvessels were identified in the infarcted heart using the fluorescent microsphere method for regional blood flow assessment 2 weeks following transplantation. There was a significant decrease in blood flow to the infarcted and peri-infarcted zone and an increase to $48 \%$ of the normal level was observed when EPCs and EPC ${ }^{\text {null }}$ treatment was induced. Notably, transplantation of $\mathrm{EPC}^{\text {DIl-4+ }}$ further increased the blood flow to $\sim 65.1 \%$ that of the normal level. However, no evident improvement was detected when treated with PBS or EPC ${ }^{\text {Dll-4- }}$ (Fig. 6).

\section{Discussion}

In terms of EPCs, abundant evidence supports their involvement in capillary growth and the formation of collateral vessels. These consequent effects have led to improved perfusion and functional recovery in animal models of myocardial and peripheral ischemia $(4,18)$. In early clinical trials, the therapeutic administration of EPCs to patients with MI or chronic angina has been associated with positive trends in perfusion (2). However, low survival and the angiogenic potential of treated cells in ischemic myocardium affects the outcome of EPC transplantation for the treatment of ischemic disease. Cell sheet grafts with genetically engineered properties to prolong stem cell survival and promote blood vessel networks integrated with pre-existing coronary may provide a potential approach to repair dead or injured myocardium (19). The present study suggests that EPCs may be efficiently transfected with lentiviral vectors encoding Dll-4 without any adverse effect on the cell viability and that

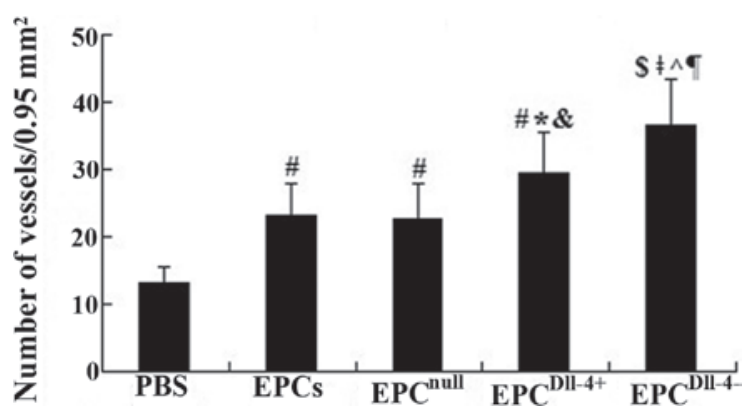

Figure 5. Immunohistochemical analysis of VIII factor-positive microvessels in the ischemic zone 2 weeks following the intravenous injection of EPCs or medium. The number of mature microvessels in the ischemia border and infarcted area was significantly increased in the EPCs, EPC null and EPC ${ }^{\text {Dll-4+ }}$ groups when compared with the PBS group. Furthermore, the vessel number was greater in the $\mathrm{EPC}^{\text {Dll-4+ }}$ group compared with the EPCs and $\mathrm{EPC}^{\text {null }}$ groups. An increased vessel was also detected in the $\mathrm{EPC}^{\text {DIl-4- }}$ group. PBS, phosphate-buffered saline; EPCs, endothelial progenitor cells; D1l-4, Delta-like-4; $\mathrm{EPC}^{\text {null }}$, EPCs infected with mock vectors; $\mathrm{EPC}^{\text {Dll-4+}}$, Dll-4-overexpressing EPCs; EPC ${ }^{\text {Dll-4- }}$, Dll-4-inhibited EPCs. ${ }^{\prime} \mathrm{P}<0.01$ vs. PBS group; ${ }^{\$} \mathrm{P}<0.001$ vs. PBS group; ${ }^{*}<0.05$ vs. EPCs group; ${ }^{\&} \mathrm{P}<0.05$ vs. EPC ${ }^{\text {null }}$ group; ${ }^{\ddagger} \mathrm{P}<0.01$ vs. EPCs group; ${ }^{\wedge} \mathrm{P}<0.01$ vs. EPC ${ }^{\text {null }}$ group; ${ }^{9} \mathrm{P}<0.01$ vs. $\mathrm{EPC}^{\mathrm{Dll}-4+}$ group.

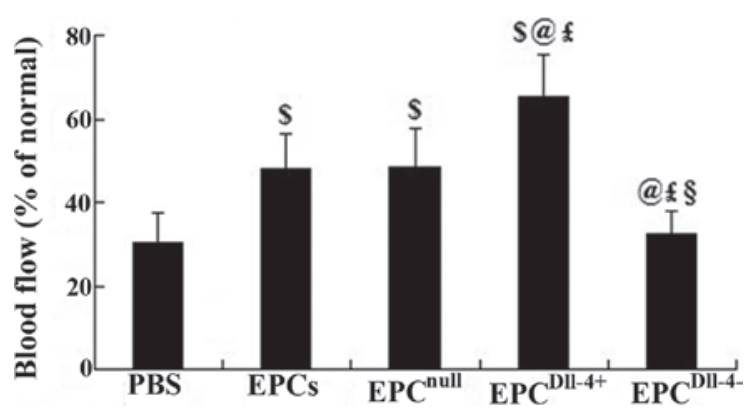

Figure 6. Measurement of blood flow in the ischemic region (including infarcted and border zone). Quantitative data suggested that $\mathrm{EPC}^{\mathrm{DIl}-4+}$ treatment significantly increased the blood flow to the ischemic tissue 2 weeks post-transplantation. EPCs, endothelial progenitor cells; EPC ${ }^{\text {null }}$, EPCs infected with mock vectors; Dll-4, Delta-like-4; EPC ${ }^{\text {Dll-4+ }}$, Dll-4-overexpressing EPCs; EPC ${ }^{\text {Dll-4- }}$, Dll-4-inhibited EPCs. ${ }^{\$} \mathrm{P}<0.001$, vs. PBS group; ${ }^{\circledR} \mathrm{P}<0.001$ vs. EPCs; ${ }^{\text {f}} \mathrm{P}<0.001$ vs. $\mathrm{EPC}^{\text {null, }}$; $\mathrm{P}<0.001$ vs. EPC ${ }^{\text {Dll-4+ }}$ group.

$\mathrm{EPC}^{\mathrm{Dll}-4+}$ exhibit a higher resistance against oxidative stress and the transfected EPCs survive for a longer period of time in the ischemic area.

Vascular network formation is coordinated by VEGF and Dll-4. Dll-4 may act downstream of VEGF as a 'brake' on VEGF-mediated angiogenic sprouting (20) and it may act to prevent overexuberant angiogenic sprouting, promoting the timely formation of a well-differentiated vascular network (21). Inhibition of Dll-4 induces the proliferation of immature vascular networks and results in poor tissue perfusion (11-13). The current in vitro study demonstrated that the number of tubular structures in the $\mathrm{EPC}^{\text {DIl-4+ }}$ group was higher compared with the EPC and $\mathrm{EPC}^{\text {null }}$ groups. In in vivo experiments, a significantly greater capacity was observed when productive vessels were formed and further increased blood flow to the infarcted and peri-infarcted zone in the groups receiving $\mathrm{EPC}^{\mathrm{DIl}-4+}$, with the exception of the group transplanted with untreated EPCs. Two weeks following cell transplantation, the cardiac function in the Dll-4-overexpressing EPCs group gradually recovered, 
whereas the PBS and untreated EPCs groups did not exhibit such effects, suggesting that tubulogenesis and enhancement of tissue perfusion played a key role in the improvement of cardiac function in MI animals. $\mathrm{EPC}^{\mathrm{Dll}-4+}$ also promotes the formation of mature vessels in the ischemic myocardium and enhances cardiac function. Thus, treated EPCs are hypothesized to be superior to the untreated EPCs. By contrast, specific knockdown of Dll-4 attenuates the ability of EPCs to form mature vascular structures in the in vitro angiogenesis assay. Injection of Dll-4-inhibited EPCs into MI animals, resulted in the formation of a dense capillary network, which appeared irregular and disorganized, thus, unable to supply ischemic myocardium with adequate perfusion. These observations were consistent with a previous study showing that Dll-4 blockade causes functional defects in angiogenesis following ischemia in mouse limp (22).

With the exception of Notch, the signaling pathways of mTOR are also closely linked to stem cell development and neovascularization. In ECs, mTOR may be necessary for EPC development since inhibition of mTOR pathways with rapamycin may lead to EPC death, which may result from inhibiting growth factor signaling (23). As an important component of cardiac tissue protection and regeneration, angiogenesis may be regulated by mTOR (24). Inhibition of mTOR may lead to a sequence of events, including elevated matrix metalloproteinase- 1 and the blockade of tissue inhibitor of metalloprotease-3 resulting in impaired angiogenesis (25). Moreover, loss of mTOR activity may also lead to a blockage of endothelial proliferation and angiogenesis (26) as well as proliferation of EPCs (23). In the current study, the expression of Notch downstream target molecule, Hey-1 and the phosphorylated status of mTOR and p70S6K (one of mTOR downstream effectors) were significantly increased under a higher expression of Dll-4 in EPCs. $\mathrm{EPC}^{\mathrm{Dll}-4+}$ transplantation also led to a similar variation as the mouse ischemic myocardium was extracted for western blot analysis. Notably, an opposite effect was observed in in vitro cultered $\mathrm{EPC}^{\text {Dll-4- }}$ and the animals treated with $\mathrm{EPC}^{\text {null }}$. A previous study by Chan et al observed that Notch signals may positively regulate the activity of the mTOR pathway in T-cell acute lymphoblastic leukemia (27). Overexpression of Dll-4 was thus hypothesized to increase Hey-1 expression via interaction with the Notch-1 or -4 receptor $(28,29)$ and in turn activate the mTOR/p70S6K-mediated angiogenesis signaling pathways.

In conclusion, the present study suggests that the intravenous injection of D11-4-overexpressing EPCs stimulates angiogenesis and tubulogenesis effectively and increases blood flow to the ischemic zone through the activation of Notch/Hey-1/mTOR/p70S6K signaling pathways. The combined strategy of EPCs transplantation with Dll-4 therapy may be proposed as a promising approach for the treatment of ischemic heart disease.

\section{References}

1. Karamysheva AF: Mechanisms of angiogenesis. Biochemistry (Mosc) 73: 751-762, 2008

2. Lawall H, Bramlage $P$ and Amann B: Treatment of peripheral arterial disease using stem and progenitor cell therapy. J Vasc Surg 53: 445-453, 2011.
3. Gimble JM, Bunnell BA and Guilak F: Human adipose-derived cells: an update on the transition to clinical translation. Regen Med 7: 225-235, 2012.

4. Kalka C, Masuda H, Takahashi T, Kalka-Moll WM, Silver M, Kearney M, Li T, Isner JM and Asahara T: Transplantation of ex vivo expanded endothelial progenitor cells for therapeutic neovascularization. Proc Natl Acad Sci USA 97: 3422-3427, 2000.

5. Asahara T, Murohara T, Sullivan A, Silver M, van der Zee R, Li T, Witzenbichler B, Schatteman G and Isner JM: Isolation of putative progenitor endothelial cells for angiogenesis. Science 275: 964-967, 1997.

6. Carmeliet P: Mechanisms of angiogenesis and arteriogenesis. Nat Med 6: 389-395, 2000.

7. Artavanis-Tsakonas S, Rand MD and Lake RJ: Notch signaling: cell fate control and signal integration in development. Science 284: 770-776, 1999.

8. Roca C and Adams RH: Regulation of vascular morphogenesis by Notch signaling. Genes Dev 21: 2511-2524, 2007.

9. Leslie JD, Ariza-McNaughton L, Bermange AL, McAdow R, Johnson SL and Lewis J: Endothelial signalling by the Notch ligand Delta-like 4 restricts angiogenesis. Development 134: 839-844, 2007.

10. Phng LK and Gerhardt H: Angiogenesis: a team effort coordinated by notch. Dev Cell 16: 196-208, 2009.

11. Scehnet JS, Jiang W, Kumar SR, et al: Inhibition of Dll4-mediated signaling induces proliferation of immature vessels and results in poor tissue perfusion. Blood 109: 4753-4760, 2007.

12. Noguera-Troise I, Daly C, Papadopoulos NJ, Coetzee S, Boland P, Gale NW, Lin HC, Yancopoulos GD and Thurston G: Blockade of Dll4 inhibits tumour growth by promoting non-productive angiogenesis. Nature 444: 1032-1037, 2006.

13. Ridgway J, Zhang G, Wu Y, et al: Inhibition of Dll4 signalling inhibits tumour growth by deregulating angiogenesis. Nature 444 : 1083-1087, 2006.

14. Liu L, Wen T, Zheng XY, Yang DG, Zhao SP, Xu DY and Lü GH: Remnant-like particles accelerate endothelial progenitor cells senescence and induce cellular dysfunction via an oxidative mechanism. Atherosclerosis 202: 405-414, 2009.

15. Chen JJ and Zhou SH: Mesenchymal stem cells overexpressing MiR-126 enhance ischemic angiogenesis via the AKT/ERK-related pathway. Cardiol J 18: 675-681, 2011.

16. Urbich C, Aicher A, Heeschen C, Dernbach E, Hofmann WK, Zeiher AM and Dimmeler S: Soluble factors released by endothelial progenitor cells promote migration of endothelial cells and cardiac resident progenitor cells. J Mol Cell Cardiol 39: 733-742, 2005.

17. Huang F, Zhu X, Hu XQ, et al: Mesenchymal stem cells modified with miR-126 release angiogenic factors and activate Notch ligand Delta-like-4, enhancing ischemic angiogenesis and cell survival. Int J Mol Med 31: 484-492, 2013.

18. Murohara T: Angiogenesis and vasculogenesis for therapeutic neovascularization. Nagoya J Med Sci 66: 1-7, 2003.

19. Deuse T, Peter C, Fedak PW, et al: Hepatocyte growth factor or vascular endothelial growth factor gene transfer maximizes mesenchymal stem cell-based myocardial salvage after acute myocardial infarction. Circulation 120 (Suppl 11): S247-S254, 2009.

20. Suchting S, Freitas C, le Noble F, Benedito R, Bréant C, Duarte A and Eichmann A: The Notch ligand Delta-like 4 negatively regulates endothelial tip cell formation and vessel branching. Proc Natl Acad Sci USA 104: 3225-3230, 2007.

21. Lobov IB, Renard RA, Papadopoulos N, Gale NW, Thurston G, Yancopoulos GD and Wiegand SJ: Delta-like ligand 4 (Dll4) is induced by VEGF as a negative regulator of angiogenic sprouting. Proc Natl Acad Sci USA 104: 3219-3224, 2007.

22. Al Haj Zen A, Oikawa A, Bazan-Peregrino M, Meloni M, Emanueli C and Madeddu P: Inhibition of delta-like-4-mediated signaling impairs reparative angiogenesis after ischemia. Circ Res 107: 283-293, 2010.

23. Miriuka SG, Rao V, Peterson M, Tumiati L, Delgado DH, Mohan R, Ramzy D, Stewart D, Ross HJ and Waddell TK: mTOR inhibition induces endothelial progenitor cell death. Am J Transplant 6: 2069-2079, 2006.

24. Maiese K, Chong ZZ, Shang YC and Hou J: FoxO proteins: cunning concepts and considerations for the cardiovascular system. Clin Sci (Lond) 116: 191-203, 2009.

25. Lemaitre V, Dabo AJ and D'Armiento J: Cigarette smoke components induce matrix metalloproteinase-1 in aortic endothelial cells through inhibition of mTOR signaling. Toxicol Sci 123: 542-549, 2011. 
26. Humar R, Kiefer FN, Berns H, Resink TJ and Battegay EJ: Hypoxia enhances vascular cell proliferation and angiogenesis in vitro via rapamycin (mTOR)-dependent signaling. FASEB J 16: 771-780, 2002.

27. Chan SM, Weng AP, Tibshirani R, Aster JC and Utz PJ: Notch signals positively regulate activity of the mTOR pathway in T-cell acute lymphoblastic leukemia. Blood 110: 278-286, 2007.
28. Shutter JR, Scully S, Fan W, Richards WG, Kitajewski J, Deblandre GA, Kintner CR and Stark KL: Dll4, a novel Notch ligand expressed in arterial endothelium. Genes Dev 14: 1313-1318, 2000

29. Hellström M, Phng LK, Hofmann JJ, et al: Dll4 signalling through Notch1 regulates formation of tip cells during angiogenesis. Nature 445: 776-780, 2007. 